\title{
Dislocation of large-diameter head metal-on-metal total hip arthroplasty and hip
} resurfacing arthroplasty

Short title: Dislocation of large-diameter head total hip arthroplasty

Simo S.A. Miettinen ${ }^{1}, \mathrm{MD}$, Tatu J. Mäkinen ${ }^{2,3}, \mathrm{PhD}$, Inari Laaksonen ${ }^{4}, \mathrm{PhD}$, Keijo Mäkelä4, PhD, Heini Huhtala ${ }^{5}$, MSc, Jukka S. Kettunen ${ }^{1}, \mathrm{PhD}$, Ville Remes ${ }^{2,3}, \mathrm{PhD}$

${ }^{1}$ Department of Orthopaedics, Traumatology and Hand Surgery, Kuopio University Hospital, P.O. Box 1777, 70211 Kuopio, Finland

2 Department of Orthopaedics and Traumatology, Helsinki University Hospital, P.O. Box 900, 00029 HUS, Finland

3 Pihlajalinna Oy, Tietokuja 4, 00330 Helsinki, Finland

4 Department of Orthopaedics and Traumatology, Turku University Hospital, P.O. Box 28, 20701 Turku, Finland

${ }^{5}$ Faculty of Social Sciences, 33014 University of Tampere, Finland

Corresponding author:

Simo Miettinen

Department of Orthopaedics, Traumatology and Hand Surgery,

Kuopio University Hospital

P.O. Box 1777

70211 Kuopio

Finland

E-mail: simo.miettinen@kuh.fi

Tel: +358 500673858

Word count: 3,293 


\section{ABSTRACT}

\section{Introduction}

Dislocation of large-diameter head (LDH) metal-on-metal (MOM) total hip arthroplasty (THA) or hip resurfacing arthroplasty (HRA) is a rare complication. This study aimed to determine the incidence and risk factors for dislocation of LDH MOM THAs and HRAs.

\section{Methods}

This retrospective analysis considered 4,038 cementless LDH MOM THAs and HRAs, 3,207 THAs in 2,912 patients and 831 HRAs in 757 patients. The end of follow-up was revision due to dislocation. Incidence of dislocation was evaluated from this study population of 4,038 , and study groups were formed. The study was designed as a case-control study, and a threefold stratified randomized control group was formed. Demographic data was collected and radiological analyses were performed in the study groups.

\section{Results}

There were $26 / 3,207(0.8 \%)$ early dislocations in the THA group, and 6/831 (0.7\%) in the HRA group $(p=0.9)$. Most LDH THA dislocations occurred in a group with head size $\leq 38$ $\mathrm{mm}(18 / 26)(p<0.001)$. In dislocated hips, there were more dysplastic acetabula and posttraumatic hips than in the control group $(p=0.036)$. In the dislocation group, the mean acetabulum component anteversion angle was $19.6^{\circ}$ (SD $13.4^{\circ}$ ) and in the control group it was $23.2^{\circ}\left(\mathrm{SD} 10.4^{\circ}\right)(p=0.006) ; 7 / 32(21.8 \%)$ of dislocated THAs needed revision surgery, and mean time to revision from the index surgery was 1.2 (SD 2.6) years. 


\section{Discussion}

Dislocations occurred more often in THAs of head size $\leq 38 \mathrm{~mm}$ and with a smaller anteversion angle of the acetabulum component. Hip dysplasia and post-traumatic osteoarthritis were more common in patients with dislocation.

Keywords: Complication total hip, Dislocation, Large-diameter head, Primary total hip arthroplasty 


\section{Introduction}

Second-generation metal-on-metal (MOM) total hip arthroplasties (THA) were introduced in the early 1990s, and they became common in the early 2000 s $(1,2)$. Large-diameter head (LDH) MOM THA was believed to be an answer to hip instability associated with smaller diameter head metal-on-polyethylene (MOP) bearing THAs (1, 3). Hip dislocation remains the most frequent complication after THA, with a reported incidence ranging from $1 \%$ to $11 \%$ with polyethylene-on-metal THAs (4-8). In LDH MOM THAs, hip dislocation rates have been found to be very low $(<1 \%)$ or even nonexistent $(1,9)$.

The main risk factors for dislocations have are female gender, advanced age, neuromuscular or cognitive disorders, substance abuse, previous hip surgery and softtissue or bone deficits of the hip (10). Hip dysplasia or technical errors in surgery (e.g. acetabulum component malposition or failure to restore the centre of hip rotation) are risk factors for dislocation $(11,12)$. High-risk patients might benefit from LDH MOM THAs in the future to avoid dislocation, even though the issues caused by adverse reaction to metal debris (ARMD) in MOM THAs have discontinued the use of MOM bearings (13).

The aims of this study were to find out the incidence of dislocation in LDH MOM THA and hip resurfacing arthroplasty (HRA), and to evaluate patients according to demographic and radiographic risk factors predisposing to hip instability. We also studied if there were any differences in the rate of dislocations between the THA and HRA components. 


\section{Methods}

A multicentre retrospective study was performed in three participating university hospitals Helsinki University Hospital, Kuopio University Hospital and Turku University Hospital. A total of 4,038 THAs were performed between January 2004 and December 2009, 3,207 THAs in 2,912 patients and 831 HRAs in 757 patients. The median follow-up time in the dislocation group was 6.1 (SD 1.5, range 2.0-7.9) years, and in the control group 4.4 (SD 1.8 , range $1.7-7.8)$ years $(p=1.0)$, respectively.

The primary outcome of this study was to find out the dislocation rate for LDH MOM hip devices, and the secondary outcome was to evaluate the possible risk factors for dislocations. Patient demographic data in terms of age, gender, preoperative diagnosis (primary osteoarthritis, developmental dysplasia of the hip, fracture, rheumatoid arthritis, avascular necrosis and other arthritis) and underlying systemic diseases affecting risk of dislocation (rheumatoid arthritis, alcohol abuse, neurological disease) were collected from the patient medical records (Table 1 ). Head size $\geq 38 \mathrm{~mm}$ were considered as LDH hip device. There were 4,038 hips including 831 HRAs and 3,207 THAs. In the THA group there were 122 THAs with head size $<38 \mathrm{~mm}$.

There was a total of 32/4,038 (0.8\%) dislocations. For these patients, a control group of threefold the number of patients was formed by selecting THAs and HRAs from the study population of 4,006 THAs and HRAs without dislocation. Patients in the control group were stratified by age, gender, hospital and by type of arthroplasty (THA or HRA). Selection was performed by assigning a different number to each THA and HRA. A random number generator was used to randomly assign THAs and HRAs to the control group. Similarity of 
randomized groups was controlled by comparing age, gender, THA components and followup time of the randomized group (96 patients) to the study population without THA dislocation $(n=3,900)$. There were no statistically significant differences in these factors between the control group and study group.

There were four different THAs and four HRAs in the dislocation group. THAs and HRAs were performed according to the manufacturers' instructions.

\section{Radiological analysis}

Radiological analyses were performed on plain radiographs taken before surgery and at the 3-month follow-up visit or at the time dislocation first occurred (Tables 2 and 3). Numerous different radiographic measurements have been used in assessing hip dysplasia at skeletal maturity $(14,15)$. For this study, we selected some of the most commonly used and repeatable measurements, assessed from the preoperative radiographs (Figures 1 and 2).

Crowe classification was used to define developmental hip dysplasia (DDH) (16). Crowe type I has $<50 \%$ subluxation, type II has between $50 \%$ and $74 \%$ subluxation, type III has between $75 \%$ and $99 \%$ subluxation and type IV has complete dislocation (16). The Wiberg centre-edge (CE) angle was measured (16). A CE angle $<20^{\circ}$ has been postulated as indicating hip dysplasia, and one $>25^{\circ}$ as indicating a normal hip $(17,18)$. The acetabular depth-width ratio (ADR) was measured along a line running perpendicularly from the width line to the deepest point of the medial arch (19) (Figure 2). For ADR, mean cut-off values are 0.235 for males and 0.233 for females (19). The femoral head extrusion index (FHEI) was measured to assess the degree of femoral head lateralization over the acetabular edge 
(20) (Figure 2). The normal range of FHEI was originally $70-100 \%$ but subsequently a cutoff value of $75 \%$ was proposed $(19,20)$.

The functional structure of the acetabulum changes due to osteoarthritis. It has been stated that fixation of the cementless acetabular component is related to the bone structure of the acetabulum (21). We classified acetabula into three types (Type A, Type B and Type C) based on their acetabulum roof morphology on plain preoperative radiographs according to Dorr (21). A Type A acetabulum has an isosceles triangle with equal medial and lateral walls or beams and a shorter base. Type B has an extended triangle which has a pseudopod that extends into the teardrop and creates a thick medial wall. Type $\mathrm{C}$ is found only in dysplastic hips, and has a right-angled triangle with a straight lateral wall; the femoral head may or may not be located under the triangle (21).

Restoration of leg length and femoral offset are important factors for the success of THA $(22,23)$. The radiographic teardrop in $A P$ view was a landmark for many of the measurements used in our study $(14,15,24)$. The inter-teardrop line was used as the transverse axis of the pelvis (Figure 1). Leg length discrepancy (LLD) was evaluated from the preoperative and postoperative radiographs, where LLD was the perpendicular distance between a line passing through the lower edge of the inter-teardrop line to a line passing through the tip of the lesser trochanter (Figure 1) $(25,26)$. Hip offset was measured with a standard method, where the offset is the perpendicular distance between the centre of rotation of the femoral head and the femoral shaft midline (Figure 1) (27). The anatomic hip centre was located from the pre- and postoperative radiographs by a method described by Fessy (28), which has been shown to be the most precise method for determination of the 
anatomic hip centre (15). Neck-femoral shaft angle was also measured from the preoperative radiographs.

Acetabular component inclination and anteversion angles were measured from the postoperative radiographs. The inclination angle of the acetabulum component was measured according to the method described by Widmer (29). Anteversion was measured according to the method described by Murray (30). The number of acetabular components which were positioned within the safe zone (5-25 anteversion and $30-50^{\circ}$ inclination) described by Lewinnek was analysed (31).

Acetabulum components were divided into four groups based on the amount of containment of the cup, as described by Sarmiento (32). Containment was measured from the postoperative radiographs. Containment by bone was recorded as 100\%, 90-99\%, 75-$99 \%$ and $<75 \%$. Radiolucent gaps on the initial postoperative radiograph, and radiolucent lines or osteolysis at the acetabular bone-acetabular component interface on subsequent radiographs were recorded according to the assessment described by DeLee (33).

Picture archiving and communication systems (PACS) were used in every participating hospital. At Helsinki University Central Hospital, there was an Agfa IMPAX (ver. 6.5.2.657) PACS, and Sectra Workstation IDS7 (ver. 15.1.8.5) PACS were used at Kuopio University hospital and Turku University Central Hospital.

\section{Intra- and interobserver error}

Measurements were re-analysed after 2 months by the same observer (S.M.) to determine intraobserver error, and by the other observer (J.K.) to determine interobserver error. Bland- 
Altman analysis was used to determine whether intra- and interobserver agreement was acceptable. Mean values and variances were tested with paired t-test and Pitman's test for differences in variance.

\section{Statistical analysis}

Comparisons between the groups were performed with Pearson's chi-square test. Comparison of continuous data was carried out using a Mann-Whitney U-test. Two-tailed $p$ values are reported, and $p<0.05$ was considered statistically significant. All the data was analysed statistically using SPSS (IBM Corp. Released 2012. IBM SPSS Statistics for Windows, Version 21.0. Armonk, NY: IBM Corp).

\section{Ethics}

The ethical review committee of the University of Turku gave permission (ETMK: 78/1801/2013) for this study.

\section{Results}

There were 26/3,207 (0.8\%) dislocations in the LDH MOM THA group and 6/831 (0.7\%) in the HRA group $(p=0.9)$. In the group of 3,207 THAs with head size $<38 \mathrm{~mm}, 5 / 122(4.1 \%)$ dislocations occurred, and 21/3,085 (0.7\%) with head size $\geq 38 \mathrm{~mm}$, respectively. There was a total of $7 / 4,038(0.2 \%)$ THA dislocations which needed revision surgery, and of these, $6 / 3,207(0.2 \%)$ occurred in the THA group and $1 / 831(0.1 \%)$ in the HRA group $(p=0.680)$. Median time to revision for any reason in the dislocation group was 42 (SD 2.2, min 1 day, max 7.0 years) days, and in the control group was 114 (SD 0.5, min 22 days, max 1.5 years) days $(p=0.635)$. The mean age of the patients in the dislocation group was 59.5 (SD 8.5, 
37.0-80.0) years, and in the control group was 59.1 (SD 9.0, 37.0-79.0) years $(p=0.956)$. Demographic data and surgical details are given in Table 1.

In Crowe DDH classification, there were no statistically significant differences between the groups (Table 3). Radiographic measurements showed that CE angle, neck-femoral shaft angle, FHEI and ADR were similar in both groups (Table 2). The rotation centre of the hip was measured with the X-Y-coordinate model descripted by Fessy (28), and there were no differences between the groups (Table 3). The change in hip offset was not statistically significant between the groups (Table 3). Acetabulum bone structure had a statistically significant difference between the groups, and there were more Type A patients in the dislocation group (Table 2). There were no statistically significant differences between the groups for LLD or LLD change in pre- and postoperative radiographs.

For postoperative radiographic measurements, the only statistically significant difference was the acetabulum component anteversion angle, which was smaller in the dislocation group $\left(19.6^{\circ}\right.$; SD 13.4 , range $\left.-12.0-40.0^{\circ}\right)$ than in the control group $\left(23.2^{\circ}\right.$; SD 10.4 , range 3.0-58.0 $)(p=0.006)$ (Table 3). Acetabulum components were positioned in Lewinnek's safe zone only in 13/32 (40.6\%) of THAs in the dislocation group and in 40/96 (41.7\%) of THAs in the control group $(p=0.917)$ (Table 3$)$. However, only one acetabulum component of a dislocated and revised THA was in Lewinnek's safe zone (Table 5).

Of the dislocated THAs, 7/32 (21.8\%) needed revision surgery, and mean time to revision from the index surgery was 1.2 (SD 2.6, min 3 days, max 7.0 years) years (Table 4). 5/7 (71\%) of the revisions due to dislocation were done within 2 months after the index surgery (Table 5), and 6/7 (86\%) of these patients had a posterolateral approach. Only one of the 
dislocated and revised HRAs and only one revised LDH THA had a head size over $38 \mathrm{~mm}$ (Table 5).

\section{Intra- and interobserver error}

Intra- and interobserver measurements were at an acceptable level. Pitman's test revealed that there were no significant differences in intraobserver measurements $(p>0.05)$.

\section{Discussion}

Dislocation is an unfortunately frequent and serious complication following conventional THA, but is extremely rare with LDH MOM THAs or HRAs $(1,4)$. Prevalence of dislocation has been reported as being between $0.3 \%$ and $10 \%$ in primary THAs (34). In a large registrybased study, revision rate due to dislocation of different head size THA was $\leq 2.5 \%$ at 12 years follow-up (35). Understanding the mechanisms and causes of dislocation are essential in clinical decision-making before and during THA. Known risk factors for THA dislocation are surgical approach and technique, operative diagnosis, arthroplasty type, hip anatomy and patient compliance and diseases (36). Dislocation causes significant costs, and it has been calculated that post-THA instability represents $10 \%$ of all revision surgeries (37).

Patient-related risk factors must be evaluated and considered preoperatively, intraoperatively and post-operatively (9). There is evidence that age is an independent risk factor for dislocation but evidence of this is still controversial (38). Advanced age (> 75 years) is related to decline in muscular tone, inability to follow postoperative protocols and higher incidence of cognitive problems which have been linked to increased dislocation rates (3941). In our study, mean patient age in the dislocation group was quite low; because of that, 
age was stratified while the control group was selected. Patients with neuromuscular and cognitive disorders as well as alcoholism have been found to be at higher risk for dislocation $(36,42)$. In our study, only two of the dislocations occurred in alcohol abusers.

Surgical factors affecting risk of dislocation are surgical approach, implant-related factors, soft-tissue repair and surgeon experience. Higher dislocation rates have been reported for the posterior approach (2.03-5.8\%) compared to the anterolateral approach (2.18-2.3\%) and to the direct lateral approach (0.55\%) (43). In our study, we did not find any statistically significant difference between surgical approaches; however, in dislocated and revised THAs there were more cases of posterolateral approach than of direct lateral approach.

Implant-related risk factors are implant type, design and head size. Larger head sizes have been found to decrease the rate of dislocation for all surgical approaches (44). A prospective study assessing three different head size THAs $(<36 \mathrm{~mm}, 36 \mathrm{~mm}$ and $>36 \mathrm{~mm})$ showed that dislocation rates were significantly lower in the group of head size $>36 \mathrm{~mm}(0 \%)$ compared to the group of head size $<36 \mathrm{~mm}(1.25 \%)$ (45). Our results are comparable to those of Allen et al., as in our study 18/26 (69.2\%) of the dislocations occurred in LDH THA of head size $\leq 38 \mathrm{~mm}$, and only one revised THA due to dislocation had a head size over $38 \mathrm{~mm}$. In a recent large sample size register study, where different bearings (ceramic-onceramic, metal-on-metal and metal-on-polyethylene) were compared, Shah et al. showed that between head sizes of $28 \mathrm{~mm}$ and $32 \mathrm{~mm}$ there was no difference in the risk of revision due to dislocation (46). Our study findings support this finding, as the highest percentage of dislocations were in THAs with head size $<38 \mathrm{~mm}$. LDH MoM bearings give excellent stability but can entail some serious problems such as ARMD, and the use of these implants is no longer recommended (13). Alternatives to LDH MoM THAs are dual mobility implants 
and constrained acetabulum liners. Dual mobility THAs have been challenging LDH MoM THAs and have been gaining popularity in recent years, mainly due to the low rate of dislocation (1.1\%) and good overall survivorship, especially in patients with a fracture of the femoral neck (47).

Forces affecting a reconstructed hip joint have been noted to alter if the rotation centre of the hip changes, and this may predispose THA to dislocation $(11,48)$. In our study, neither preoperative nor postoperative radiological measurements showed any statistically significant difference between the groups; in addition, dislocation group measurements were within normal limits given in the literature $(17,19,20)$. Changes in hip rotation centre and hip offset between preoperative and postoperative measurements were not statistically significant. LLD change was also at an acceptable level, and there were no statistically significant differences between preoperative and postoperative measurements in either group in our study. It is more demanding to perform THA on a dysplastic hip joint than on normal hip anatomy, and further on, DDH raises the risk of dislocation $(12,16)$. In our study, we did not find any statistically significant differences between the groups for DDH measurements. However, when comparing operative diagnosis, there were more dysplastic hip joints in the dislocation group.

Component malposition is the most common cause of instability following THA. Acetabular component placement has been deemed suboptimal if outside Lewinnek's safe zone (31). Excessive anteversion may result in anterior dislocation, and excessive retroversion may result in posterior dislocation. The most common surgical error is acetabular component placement in excessive anteversion and abduction (48). In our study, dislocation occurred more often in THAs where the anteversion angle was smaller than in the control group. 
However, in both groups anteversion and inclination angle were within Lewinnek's safe zone. In addition, the mean difference of anteversion angles in between-group comparison was small $\left(3.6^{\circ}\right)$, which makes the clinical relevance of the difference non-significant. Acetabulum components were out of Lewinnek's safe zone in 6/7 of the revised and dislocated THAs. It can be assumed that technical error in acetabulum component positioning in the index surgery is the main reason for revision in cases where dislocation has occurred soon after the index surgery. There was more acetabulum roof morphology variation and more dysplastic hips in the dislocation group, which might contribute to technical error and acetabulum component malpositioning.

Most single-episode dislocations of THA can be treated successfully conservatively, as our study showed. Most dislocations occur early (0-6 months) after the index surgery, and lead more often to revision surgery $(34,49)$. Our study finding supports these previous study findings, as $5 / 7$ of these revisions were done within 2 months after the index operation.

The retrospective study design has some inherent limitations, which might be minimized by a prospective study design. Typical flaws of retrospective studies also concern our study. The influence of postoperative activity and range of motion restrictions on hip dislocation after THA are controversial, and that is why we did not include those factors in our study $(40,41)$. Surgeon experience affects the risk of dislocation. Surgeons who do fewer than 30 THAs yearly have the highest dislocation rates (50). We did not study surgeons' experience because there was a total of 46 surgeons in three participating hospitals. These surgeons performed 4,038 THAs, and it was not possible to determine the yearly amount of THAs for each surgeon from the hospitals' medical records. Also, surgical technique between orthopaedic surgeons may not be reproducible, and this causes differences between 
surgeons' personal results. In our study there were no revisions due to ARMD and one reason for this might be the short follow-up as, typically, ARMD reactions appear during a longer follow-up. A history of spinal fusion has been found to be an independent risk factor for dislocation (51). However, in this study we did not explore this as a risk factor because our assumption was that there are only a few spinal fusion patients in this small sample size of a dislocation group $(n=32)$, rendering the statistical and clinical significance inconclusive.

We showed that dislocation of LDH THA is a very rare complication. Hip dysplasia was associated with dislocation even with larger head sizes. Placing the acetabulum component at the correct inclination and anteversion angles, and restoring the hip anatomical rotation centre reduces the risk of dislocation. In our study, dislocations occurred more often in THAs where acetabulum component anteversion angles were smaller. In the past, LDH MoM bearings were thought to be an answer for hip instability related to smaller head size THAs. However, due to ARMD issues, these implants are banned. Nevertheless, some small minority of patients with a high risk of dislocation (e.g. an alcohol abuser with an acute femoral neck fracture) and who have contraindication to dual mobility implant or constrained acetabular liner, might be considered to have LDH MOM THA to reduce the risk of dislocation, even though it is not generally advisable. 


\section{Acknowledgements}

This study was made possible by support from the Finnish Arthroplasty Society, Finnish Research Foundation for Orthopedics and Traumatology, Research Foundation of Kuopio University Hospital, Finnish Cultural Foundation: North Savo Regional fund, The Finnish Medical Foundation Duodecim and Foundation of Vappu Uuspää.

\section{Declaration of conflicting interests}

Each author certifies that he or she has no commercial associations that might pose a conflict of interest in connection with the submitted article.

\section{Authors contributions}

S.M. participated in planning the study protocol, collecting data for this study at Kuopio University Hospital, analyzing radiographs and statistics, and preparing the manuscript.

T.M. participated in planning the study protocol, collecting data for this study at Helsinki University Hospital, and preparing the manuscript.

I.K. participated in planning the study protocol, collecting data for this study at Turku University Central Hospital, and preparing the manuscript.

K.M. participated in planning the study protocol, collecting data for this study at Turku University Central Hospital, and preparing the manuscript.

H.H. participated in planning the study protocol, performing statistical analyses, and preparing the manuscript. 
J.K. participated in planning the study protocol, collecting data for this study at Kuopio University Hospital, and preparing the manuscript.

V.R. participated in planning the study protocol, collecting data for this study at Helsinki University Hospital, and preparing the manuscript. 


\section{References}

1. Jameson SS, Lees D, James P. Lower rate of dislocation with increased femoral head size after primary hip replacement: a five-year analysis of NHS patients in England. J Bone Joint Surg Br 2011; 93: 876-880.

2. Malviya A, Ramaskandhan J, Holland JP, Lingard EA. Current Consepts review. Metal-on-Metal Total Hip Arthroplasty. J Bone Joint Surg Am 2010; 92: 1675-83.

3. Girard J, Kern G, Migaud H, Delaunay C, Ramdane N, Hamadouche M. Primary total hip arthroplasty revision due to dislocation: prospective French multi-center study. Orthop Traumatol Surg Res 2013; 99: 549-553.

4. Bozic KJ, Kurtz SM, Lau E, Ong K, Vail TP, Berry DJ. The epidemiology of revision total hip arthroplasty in the United States. J Bone Joint Surg Am 2009; 91: 128-133.

5. Dobzyniak M, Fehring TK, Odum S. Early failure in total hip arthroplasty. Clin Orthop Relat Res 2006; 447: 76-78.

6. Dorr LD, Wolf AW, Chandler R, Conalty JP. Classification and treatment of dislocations of total hip arthroplasty. Clin Orthop 1983; 173: 151-8.

7. Hedlundh $\mathrm{U}$, Sanzen L, Fredin $\mathrm{H}$. The prognosis and treatment of dislocated total hip arthroplasties with a 22 mm head. J Bone Joint Surg Br 1997; 79: 374-8.

8. Turner RS. Postoperative total hip prosthetic femoral head dislocations. Incidence, etiologic factors, and management. Clin Orthop 1994; 301: 196-204.

9. Tao R, Liu F, Liu YK, Lu Y, Xu H, Cao Y, Zhou ZY, Wang W. A prospective comparative study of hip resurfacing arthroplasty and large-diameter head metal-onmetal total hip arthroplasty in younger patients-a minimum of five year follow-up. Int Orthop. 2018. Epub ahead of print. 
10. Werner BC, Brown TE. Instability after total hip arthroplasty. World J Orthop 2012; 3: 122-130.

11. Lecerf G, Fessy MH, Philippot R, Massin P, Giraud F, Flecher X, Girard J, Mertl P, Marchetti E, Stindel E. Femoral offset: anatomical concept, definition, assessment, implications for preoperative templating and hip arthroplasty. Orthop Traumatol Surg Res. 2009; 95: 210-9.

12. Brown TD, Elkins JM, Pedersen DR, Callaghan JJ. Impingement and dislocation in total hip arthroplasty: mechanisms and consequences. Iowa Orthop J. 2014; 34: 115.

13. Medicines and Healthcare products Regulatory Agency (MHRA). Medical device alert: all metal-on-metal (MoM) hip replacements, 2010 (MDA/2010/ 03). http://www.mhra.gov.uk/home/groups/dts-bs/documents/medicaldevicealert/ con079162.pdf. Date last accessed 21 Feb 2017.

14. Laborie LB, Lehmann TG, Engesæter I $\varnothing$, Eastwood DM, Engesæter LB, Rosendahl K. Prevalence of radiographic findings thought to be associated with femoroacetabular impingement in a population-based cohort of 2081 healthy young adults. Radiology 2011; 260: 494-502.

15. Schofer MD, Pressel T, Heyse T, Schmitt J, Boudriot U. Radiological determination of the anatomic hip center from pelvic landmarks. Acta Orthopaedica Belgica 2010; 76: $479-485$.

16. Crowe JF, Mani VJ, Ranawat CS. Total hip replacement in congenital dislocation and dysplasia of the hip. J Bone Joint Surg Am 1979; 61-A: 15-23.

17. Wiberg G. Studies on dysplastic acetabula and congenital subluxation of the hip joint with special reference to the complication of osteoarthritis. Acta Chir Scand. 1939; 83: 7-135. 
18. Cooperman D. What is the Evidence to support Acetabular Dysplasia as a Cause of Osteoarthritis? J Pediatr Orthop 2013; 33; S2-S7.

19. Cooperman DR, Wallensten R, Stulberg SD. Acetabular dysplasia in the adult. Clin Orthop Relat Res. 1983; 175: 79-85.

20. Mast NH, Impellizzeri F, Keller S, Leunig M. Reliability and agreement of measures used in radiographic evaluation of the adult hip. Clin Orthop Relat Res. 2011; 469: 188-99.

21. Dorr LD, Bechtol CO, Watkins RG, Wan Z. Radiographic anatomic structure of the arthritic acetabulum and its influence on total hip arthroplasty. J Arthroplasty 2000; 15: 890-900.

22. Asayama I, Naito M, Fujisawa M, Kambe T. Relationship between radiographic measurements of reconstructed hip joint position and the Trendelenburg sign. $\mathrm{J}$ Arthroplasty 2002; 17: 747-751.

23. Clark CR, Huddleston HD, Schoch EP 3rd, Thomas BJ. Leg-length discrepancy after total hip arthroplasty. J Am Acad Orthop Surg. 2006; 14: 38-45

24. Vare VBJ. The anatomy of the pelvic tear figure. J Bone Joint Surg Am 1952; 34: 167-9.

25. Kjellberg M, Al-Amiry B, Englund E, Sjödén GO, Sayed-Noor AS. Measurement of leg length discrepancy after total hip arthroplasty. The reliability of a plain radiographic method compared to CT-scanogram. Skeletal Radiol 2012; 41: 187-91

26. Meermans G, Malik A, Witt J, Haddad F. Preoperative radiographic assessment of limb-length discrepancy in total hip arthroplasty. Clin Orthop Rel Res 2010; 469: 1677-1682. 
27. Woolson ST, Hartford JM, Sawyer A. Results of a method of leg-length equalization for patients undergoing primary total hip replacement. J Arthroplasty 1999; 14: 159164.

28. Fessy MH, N'diaye A, Carret JP, Fischer LP. Locating the center of rotation of the hip. Surg Radiol Anat 1999; 21: 247-250.

29. Widmer KH. A simplified method to determine acetabular cup anteversion from plain radiographs. J Arthroplasty 2004; 19: 387- 90.

30. Murray DW. The definition and measurement of acetabular orientation. J Bone Joint Surg (Br) 1993; 75: 228-232.

31. Lewinnek GE, Lewis JL, Tarr R, Compere CL, Zimmerman JR. Dislocations after total hip-replacement arthroplasties. J Bone Joint Surg Am 1978; 60: 217-220.

32. Sarmiento A, Ebramzadeh E, Gogan WJ, McKellop HA. Cup containment and orientation in cemented total hip arthroplasties. J Bone Joint Surg Br 1990; 72-B: 9961002.

33. DeLee JG, Charnley J. Radiological demarcation of cemented sockets in total hip replacement. Clin Orthop Relat Res. 1976; 121: 20-32.

34. Parvizi J, Picinic E, Sharkey PF. Revision total hip arthroplasty for instability: surgical techniques and principles. J Bone Joint Surg Am 2008; 90: 1134-42.

35. Kostensalo I, Junnila M, Virolainen P, Remes V, Matilainen M, Vahlberg T, Pulkkinen $P$, Eskelinen A, Mäkelä KT. Effect of femoral head size on risk of revision for dislocation after total hip arthroplasty: a population-based analysis of 42,379 primary procedures from the Finnish Arthroplasty Register. Acta Orthop. 2013; 84: 342-7.

36. Soong M, Rubash HE, Macaulay W. Dislocation after total hip arthroplasty. J Am Acad Orthop Surg. 2004; 12: 314-321. 
37. Girard J, Lavigne M, Vendittoli PA, Roy AG. Biomechanical reconstruction of the hip joint: a randomized study comparing total hip resurfacing and total hip arthroplasty. J Bone Joint Surg Br. 2006; 6: 721-726.

38. Sanchez-Sotelo J, Berry DJ. Epidemiology of instability after total hip replacement. Orthop Clin North Am. 2001; 32: 543-552.

39. Hailer NP, Weiss RJ, Stark A, Karrholm J. The risk of revision due to dislocation after total hip arthroplasty depends on surgical approach, femoral head size, sex, and primary diagnosis. An analysis of 78,098 operations in the Swedish Hip Arthroplasty Register. Acta Orthop 2012; 83: 442-448.

40. Jørgensen CC, Kjaersgaard-Andersen P, Solgaard S, Kehlet H. Hip dislocations after 2,734 elective unilateral fast-track total hip arthroplasties: incidence, circumstances and predisposing factors. Arch Orthop Trauma Surg. 2014; 134: 1615-22.

41. Sharma V, Morgan PM, Cheng EY. Factors influencing early rehabilitation after THA: a systematic review. Clin Orthop Relat Res 2009; 467: 1400-1411.

42. Fackler CD, Poss R. Dislocation in total hip arthroplasties. Clin Orthop Relat Res. 1980; 151: 169-178.

43. Patel PD, Potts A, Froimson MI. The dislocating hip arthroplasty: prevention and treatment. J Arthroplasty. 2007; 22: 86-90.

44. Berry DJ, von Knoch M, Schleck CD, Harmsen WS. Effect of femoral head diameter and operative approach on risk of dislocation after primary total hip arthroplasty. $\mathrm{J}$ Bone Joint Surg Am 2005; 87: 2456-2463.

45. Allen CL, Hooper GJ, Frampton CMA. Do larger heads improve the functional outcome in total hip arthroplasty? J Arthroplasty 2014; 29: 401-404.

46. Shah SM, Walter WL, Tai SM, Lorimer MF, de Steiger RN. Late Dislocations After Total Hip Arthroplasty: Is the Bearing a Factor? J Arthroplasty. 2017; 32: 2852-56. 
47. Darrith B, Courtney PM, Della Valle CJ. Outcomes of dual mobility components in total hip arthroplasty: a systematic review of the literature. Bone Joint J. 2018; 100B: 11-19.

48. Kennedy JG, Rogers WB, Soffe KE, Sullivan RJ, Griffen DG, Sheehan LJ. Effect of acetabular component orientation on recurrent dislocation, pelvic osteolysis, polyethylene wear, and component migration. J Arthroplasty 1998; 13: 530-534.

49. Berry DJ, von Knoch M, Schleck CD, Harmsen WS. The cumulative long-term risk of dislocation after primary Charnley total hip arthroplasty. J Bone Joint Surg Am 2004; 86: 9-14.

50. Hedlundh U, Ahnfelt L, Hybbinette $\mathrm{CH}$, Weckstrom J, Fredin H. Surgical experience related to dislocations after total hip arthroplasty. J Bone Joint Surg Br. 1996; 78-B: 206-209.

51. Gausden EB, Parhar HS, Popper JE, Sculco PK, Rush BNM. Risk Factors for Early Dislocation Following Primary Elective Total Hip Arthroplasty. J Arthroplasty. 2018. Epub ahead of print. 
Tables

Table 1. Demographic data of the patients.

\begin{tabular}{|c|c|c|c|}
\hline & $\begin{array}{l}\text { Dislocation } \\
\text { group } \\
\text { n (\%) }\end{array}$ & $\begin{array}{l}\text { Control } \\
\text { group } \\
\text { n (\%) }\end{array}$ & \multirow[b]{2}{*}{$\begin{array}{l}p- \\
\text { value }\end{array}$} \\
\hline & & & \\
\hline Gender & & & 0.757 \\
\hline Male & $19(59.4)$ & $54(56.3)$ & \\
\hline Female & $13(40.6)$ & $42(43.8)$ & \\
\hline Surgical approach & & & 0.707 \\
\hline Posterior & $26(81.3)$ & $75(78.1)$ & \\
\hline Direct lateral (Hardinge) & $6(18.8)$ & $21(21.9)$ & \\
\hline Operation side & & & 0.759 \\
\hline Left & $17(53.1)$ & $48(50.0)$ & \\
\hline Right & $15(46.9)$ & $48(50.0)$ & \\
\hline Operation diagnosis & & & 0.046 \\
\hline Primary osteoarthritis & $16(50.0)$ & $70(72.9)$ & \\
\hline Developmental dysplasia of the hip & $3(9.4)$ & $10(10.4)$ & \\
\hline Fracture (acute or sequelae of the & & & \\
\hline hip) & $5(15.6)$ & $4(4.2)$ & \\
\hline Rheumatoid arthritis & $3(9.4)$ & $2(2.1)$ & \\
\hline
\end{tabular}


Avascular necrosis

Other arthritis

Diseases affecting risk of dislocation

None

Rheumatoides arthritis

Other arthritis

Long term cortisone medication use

Osteoporosis

Alcohol abuse

Neurological disease

THA type

HRA

Conventional THA

Conventional THA subtypes

Head diameter $>38 \mathrm{~mm}$

Head diameter 38mm

Head diameter $<38 \mathrm{~mm}$

Femoral implant type

Tapered

Fit and fill

Other

HRA

Intraoperative complication
$4(12.5)$

$8(8.3)$

$1(3.1)$

$2(2.1)$

$27(84.4)$

$87(90.6)$

$2(6.3)$

$2(2.1)$

$1(3.1)$

$0(0.0)$

$0(0.0)$

$2(2.1)$

$0(0.0)$

$1(1.0)$

$2(6.3)$

$1(1.0)$

$0(0.0)$

$3(3.1)$

1.000

$6(18.8 .6)$

18 (18.8)

$26(81.3)$

78 (81.3)

$<0.001$

8 (25.0)

61 (63.5)

13 (40.6)

15 (15.6)

5 (15.6)

2 (2.1)
0.900

1 (3.1)

5 (5.2)

25 (78.1)

72 (75.0)

$0(0.0)$

$1(1.0)$

6 (18.8)

18 (18.8)

27 (84.4)

0.390

90 (93.8) 
Periprosthetic fracture

$3(9.4)$

$3(3.1)$

Nerve injury

$1(3.1)$

2 (2.1)

Other

$1(3.1)$

$1(1.0)$ 
Table 2. Radiographic assessments.

\section{Dislocation group Control group}

$\mathrm{n}=32 \quad \mathrm{n}=96 \quad \begin{aligned} & p- \\ & \text { valu }\end{aligned}$

\section{Preoperative radiographic}

measurements

41.9 (SD 13.195, range 41.3 (SD 12.885, range 0.16

\begin{tabular}{|c|c|c|c|}
\hline Center-Edge angle (CE) & $3.0-74.0)$ & $14.0-76.0)$ & 4 \\
\hline & 136.0 (SD 5.734, range & 135.5 (SD 5.058, range & 0.16 \\
\hline Neck-femoral shaft angle & $124.0-149.0)$ & $126.0-150.0)$ & 9 \\
\hline & 0.255 (SD 0.069 , range & 0.254 (SD 0.056, range & 0.67 \\
\hline Acetabulum depth ratio & $0.13-0.48)$ & $0.11-0.42)$ & 6 \\
\hline Femoral head extrusion & 81.3 (SD 13.881, range & 81.3 (SD 12.373, range & 0.64 \\
\hline$x(F H E I)$ & $49.0-100.0)$ & $54.2-100.0)$ & 4 \\
\hline & 31.9 (SD 6.552, range & 33.6 (SD 5.856, range & 0.64 \\
\hline Fessy $\mathrm{x}, \mathrm{mm}$ & $16.2-43.2)$ & 22.5-51.3) & 2 \\
\hline & 23.8 (SD 7.618, range & 22.9 (SD 6.782, range & 0.05 \\
\hline Fessy y, mm & $10.0-41.4 .0)$ & $11.7-50.4)$ & 2 \\
\hline & 40.4 (SD 6.273, range & 40.2 (SD 8.670, range & 0.45 \\
\hline Hip offset, mm & $30-50)$ & $15-57)$ & 0 \\
\hline
\end{tabular}


37.3 (SD 9.149, range 38.2 (SD 9.086, range 0.20

LLD, mm

7-49)

17-59)

2

Postoperative radiographic

measurements

29.6 (SD 5.477, range 28.2 (SD 4.390, range 0.22

Fessy $\mathrm{x}, \mathrm{mm}$

21.6-45.0)

18.9-39.6)

9

22.8 (SD 6.143, range 22.3 (SD 5.102, range 0.60

Fessy y, mm

13.0-46.8)

13.5-49.5)

6

Change of the hip 3.33 (SD 10.429, range 6.0 (SD 8.638, range -0.50

rotation center, $\mathrm{mm}$

$-22.7-15.3)$

21.0-27.0)

2

Change of the hip

rotation center

6

$<10 \mathrm{~mm}$

$22(68.8)$

$63(65.6)$

$\geq 10 \mathrm{~mm}$

$10(31.3)$

$33(34.4)$

Unable to measure

0

48.0 (SD 8.533, range 49.1 (SD 7.375, range 0.16

Hip offset, mm 21-64) $32-65)$

7

Hip offset change 7.5 (SD 7.331, range -

8.8 (SD 6.843, range -0.65

postoperatively, $\mathrm{mm}$

1179-18.0)

$5.5-24.3)$

4

45.8 (SD 9.189, range

46.4 (SD 8.513 range 0.15

LLD, mm

34.2-62.1)

27.0-62.1)

2

LLD

change

0.15

postoperatively

$\leq 20 \mathrm{~mm}$ lengthing $\quad 22(68.8)$

$81(84.4)$ 

$>20 \mathrm{~mm}$ lengthing
$4(12.5)$
$5(5.2)$
Shortening of the leg 4 (12.5)
$6(6.3)$
Unable to measure $2(6.3)$
$4(4.2)$

Acetabular component 47.4 (SD 6.031, range 45.6 (SD 7.805, range 0.95

inclination angle

$38.0-60.0)$

Acetabular

component

anteversio angle

$-12.0-40.0)$

Cup position at Lewinnek

safe zone

No

19 (59.4)

$13(40.6)$
25.0-64.0)

3

23.2 (SD 10.443, range 0.00

$-3.0-58.0)$

6

0.91

7

$56(58.3)$

$4(41.7)$

Table 3. Radiographic classifications.

Dislocation

group

$p-$

$\mathrm{n}=\mathbf{3 2}$
Control

group

$\mathrm{n}=96$

value

\section{radiographic}

classifications

Acetabulum bone structure

0.011

Type A

$6(18.8)$

$3(3.1)$ 
Type B

Type C

Crowe DDH classification

None

Type I

Type II

Type III

Type IV

Postoperative

measurements

Cup containment

Complete $100 \%$

90-99\%

$75-90 \%$

$<75 \%$

Radiolucent lines (>2mm)

None

Yes
$20(62.5)$

$6(18.8)$

$21(21.9)$

$72(75.0)$

0.084

$26(81.3)$

83 (86.5)

2 (6.3)

7 (7.3)

$3(9.4)$

$6(6.3)$

$1(3.1)$

0

0

0 radiographic 
Table 4. Revised patients.

\begin{tabular}{|c|c|c|c|}
\hline & $\begin{array}{l}\text { Dislocation group } \\
\text { n (\%) }\end{array}$ & $\begin{array}{l}\text { Control group } \\
\text { n (\%) }\end{array}$ & \\
\hline & & & $p$-value \\
\hline Revision & & & $<0.001$ \\
\hline Yes & $10(31.3)$ & $6(6.3)$ & \\
\hline No & $22(68.7)$ & $90(93.7)$ & \\
\hline Reason for revision & & & 0.028 \\
\hline Dislocation & 7 & 0 & \\
\hline Infection & 1 & 0 & \\
\hline Acetabulum component loosening & 1 & 1 & \\
\hline Periprosthetic fracture & 1 & 4 & \\
\hline Component malposition & 0 & 1 & \\
\hline
\end{tabular}


Table 5. Dislocation and revised patients.

\begin{tabular}{|c|c|c|c|c|c|c|c|c|}
\hline Surgical approach & Follow-up (years) & THA type & THA model & Head size & Time to revision & Inclination angle & Anteversion angle & Lewinnek's safe zone \\
\hline Posterolateral & 3.9 & Conventional THA & Synergy/BHR & 46 & 1.3 years & 39 & -2 & No \\
\hline Posterolateral & 4.4 & HRA & Biomet Recap & 48 & 36 days & 55 & $\mathrm{~N} / \mathrm{A}$ & No \\
\hline Posterolateral & 6.1 & Conventional THA & Biomet BiMetric/M2a38 & 38 & 3 days & 60 & 35 & No \\
\hline Posterolateral & 6.6 & Conventional THA & Biomet BiMetric/M2a38 & 38 & 44 days & 42 & 22 & Yes \\
\hline Posterolateral & 6.1 & Conventional THA & Biomet BiMetric/M2a38 & 38 & 7 days & 50 & 14 & No \\
\hline Posterolateral & 2.0 & Conventional THA & Corail/Summit Pinnacle & 36 & 39 days & 40 & -3 & No \\
\hline Direct lateral (Hardinge) & 7.9 & Conventional THA & Biomet BiMetric/M2a38 & 38 & 7.0 years & 48 & 34 & No \\
\hline
\end{tabular}


Figure legends

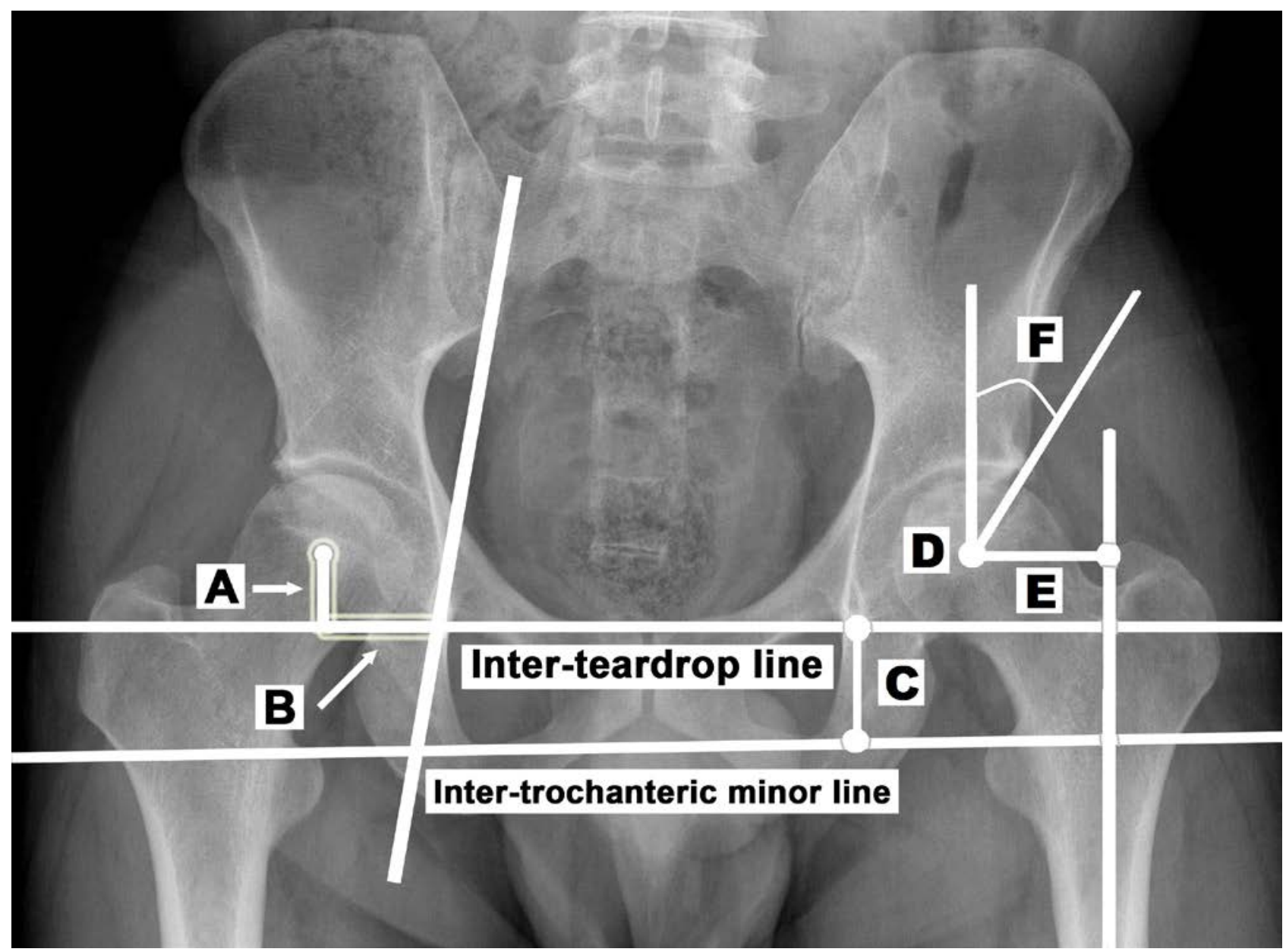

Figure 1. Vertical (A) distance of the hip joint center is measured between center of the femoral head and perpendicular to inter-teardrop line. Horizontal (B) distance of the hip joint center is measured from intersection of the distal end of the inter-teardrop line and from the line between inferior edge of the sacroiliac joint and teardrop. Leg length discrepancy (LLD) measurement (C) descripted by Kjellberg (24). D is rotation center of the hip. A standard hip offset measurement (E) descripted by Woolson (26). Center-edge angle $(F)$ is formed by vertical line through the center of the femoral head and perpendicular to the transverse axis of the pelvis. 


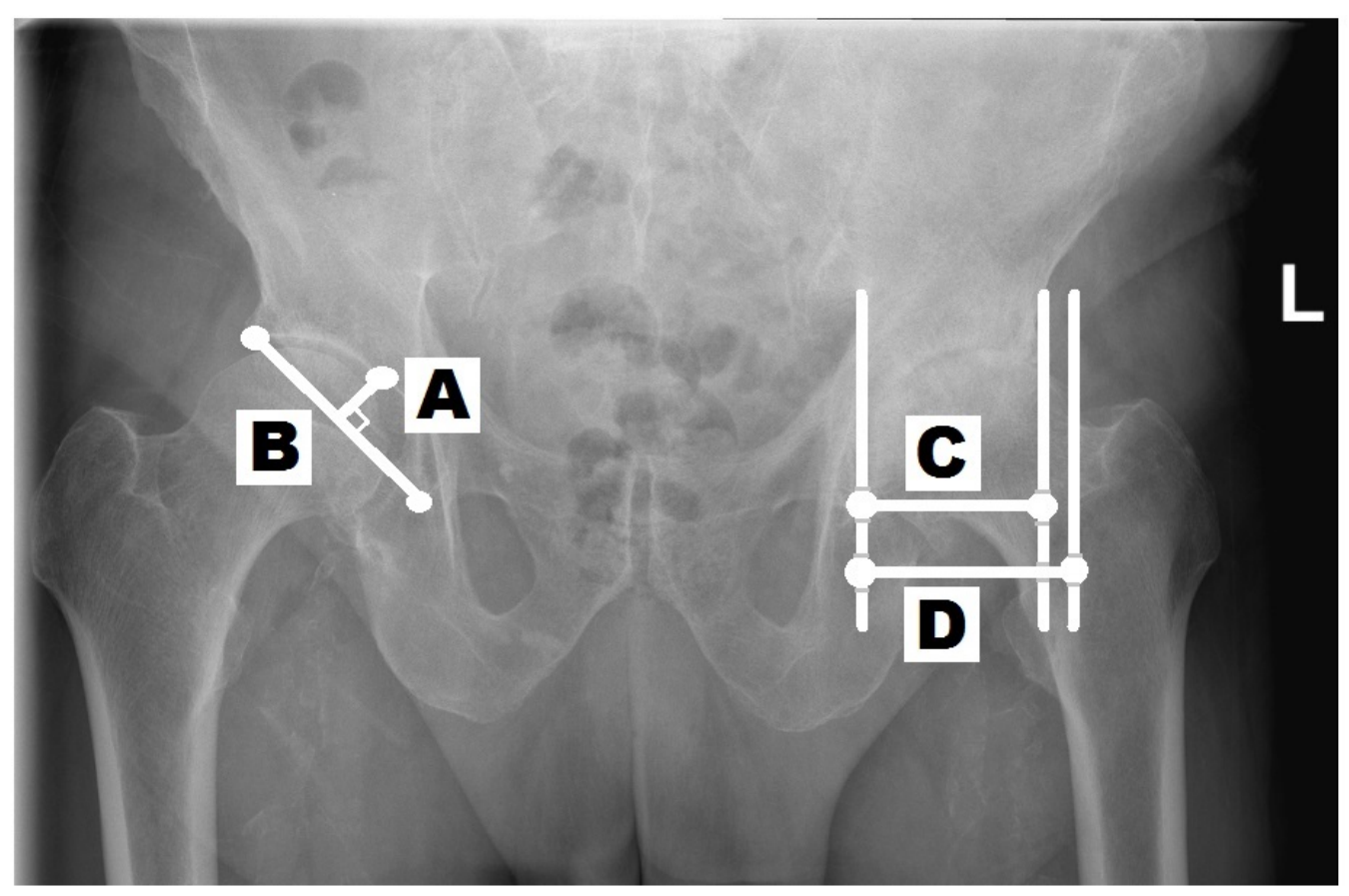

Figure 2. The acetabular depth-width ratio (ADR) is the depth of the acetabulum (A) divided by the width of the acetabulum (B). The femoral head index (FHEI) quantifies how much of the femoral head is covered by the acetabulum (C/D x 100). 